\title{
ARTICLE
}

\section{Uncertainty Analysis of the Fission Product Behaviors during Severe Accidents for a Typical PWR}

\author{
Soo Yong PARK* and Kwang Il AHN \\ Korea Atomic Energy Research Institute, Daejeon, Korea
}

\begin{abstract}
This paper illustrates an application of a severe accident analysis code, MAAP, to the uncertainty analysis of fission products (FPs) behaviors in case of severe reactor accidents. The MAAP code is a system level computer code capable of performing integral analyses of potential severe accident progressions in nuclear power plants, whose main purpose is to support Level 2 probabilistic safety assessment or severe accident management strategy developments. The code employs lots of user-options for supporting sensitivity and uncertainty analysis. The present application is mainly focused on determining an estimate of the FPs in release and transport processes and relative importance of the dominant contributors to the predicted FPs. Key modeling parameters and phenomenological models employed for the present uncertainty analysis are closely related to FP release correlations, vapor-aerosol equilibrium, vapor-surface equilibrium for a re-vaporization calculation, and aerosol decontamination factors. The Korean standardized nuclear power plant, OPR-1000, has been used as a reference plant for the analysis.
\end{abstract}

\section{KEYWORDS: severe nuclear accident, fission product behavior, uncertainty analysis, MAAP code}

\section{Introduction}

During a severe nuclear accident, an analysis of source term and relevant fission product (FP) behavior is an essential part for an accident management of nuclear power plants and an emergency planning. Relating with the behavior of FPs for severe accidents, there are several integral computer codes, such as MAAP (Modular Accident Analysis Program) $)^{1)}$ and MELCOR ${ }^{2)}$ to analyze the accident progression, thermal-hydraulic phenomena, radionuclide behavior and transport, and environmental consequence. Those codes are fundamentally a fast running tool characterized with simplified models (subjected to bestestimate models in parts), and thus they are subjected to many uncertainties, when they are practically applied to reactor cases. By the reason, they employ lots of useroptions for supporting sensitivity and uncertainty analysis in a diverse way. The MAAP code is also the case. On the other hand, key modeling parameters characterizing a source term behavior are closely related to FP release correlations, vaporaerosol equilibrium, vapor-surface equilibrium for a revaporization calculation, hygroscopic aerosol growth calculation, aerosol deposition correlation, aerosol coagulation calculation, and aerosol decontamination factors.

This paper illustrates an application of the MAAP to the uncertainty analysis of FPs behavior in case of severe reactor accidents. The present application is mainly focused on determining an estimate of the FPs in release and transport processes, i.e. (1) their release from the reactor core, (2) their distribution in a reactor coolant system (RCS), molten corium, and containment during a severe accident. Key modeling parameters employed in the FP models of the

${ }^{*}$ Corresponding Author, Tel. No: +82-42-868-8298, Fax No:. +8242-868-8256, E-Mail; sypark@kaeri.re.kr
MAAP code are taken into account as a main source of the present uncertainty analysis.

\section{Methodology}

The application was performed by using a MAAP model of OPR-1000(Optimized Power Reactor) for an estimate of the FP behaviors. For which, a small loss of coolant accident (LOCA) is simulated as an initiating event of severe accident sequence. The break size considered is about a two inches diameter in the cold leg. All the emergency core cooling systems, auxiliary feedwater system, and the containment spray are assumed to be inoperable to simulate the severe core damage scenario.

The basic approach of this methodology is to 1) identify the MAAP input parameters, sensitivity coefficients, and modeling options that describe or influence the FP behavior, 2) prescribe likelihood descriptions of the potential range of these parameters, and 3) evaluate the code predictions using a number of random combinations of parameter inputs sampled from the likelihood distributions. This method of characterizing uncertainty in reactor accident progression is similar to the method used by Randall O. Gauntt ${ }^{3)}$ where the MELCOR code was used. In order to limit the number of "realizations" (code calculations) needed to characterize the full range of uncertainty, the Latin Hypercube Sampling method (LHS) is used to sample the input parameter distributions.

The thermal hydraulic, severe accident phenomenological or radiological analyses for the evaluation have been performed by using MAAP 4.06. The code is a system level computer code capable of performing integrated analyses of severe accident progression, supporting level 2 probabilistic safety assessment studies or accident management strategy developments 
MAAP4 contains FP behavior models that calculate the release of FPs from the core and relocated core material invessel and from core debris ex-vessel. It also calculates the transport and deposition of FPs in the RCS and in the containment as well as the release of FPs to the environment through containment leakage and failure paths. The code sorts the initial masses of $22 \mathrm{FP}$ elements into 12 groups and then tracks the mass of each group in each of 4 physical states (that is, vapor, aerosol, deposited on surfaces, and contained in corium) in the various components of the RCS and containment.

In order to quantify uncertainties addressed in the MAAP code, a computer program, MOSAIQUE ${ }^{4}$, has been applied, which is recently developed by KAERI (Korea Atomic Energy Research Institute). The program is fully-automated software to quantify uncertainties addressed in the thermal hydraulic analysis models or codes.

\section{Base Case Analysis}

In advance of uncertainty analyses, a simple base case has been performed. The selected scenarios are a typical small LOCA without any recovery action. The calculation results are summarized in Table 1. The value of MAAP uncertain input parameters, which will be discussed below, for this base case is taken from the recommended value (the most likely value) of Table 2 .

Table 1. Timing of Key Events for the Small LOCA in OPR-1000

\begin{tabular}{|l|c|}
\hline Event & $\begin{array}{l}\text { Time (Base Case) } \\
\text { hours, }(\text { seconds })\end{array}$ \\
\hline SG Dryout & $0.8(2,870)$ \\
\hline Core Uncovery Start & $0.8(2,980)$ \\
\hline Cladding damage Start & $1.2(4,320)$ \\
\hline Corium Relocation Start & $2.0(7,260)$ \\
\hline Reactor Vessel Failure & $3.1(11,120)$ \\
\hline Containment Building Failure & $>72(259,200)$ \\
\hline
\end{tabular}

Among the FPs group, noble gas and cesium iodine group are considered as more volatile. The noble gas, which is chemically inert materials, poses a considerably lesser danger to human health. Instead, the iodine is one of the elements vital to the proper functioning of the human body. Iodine exits from damaged fuel rods predominantly as cesium iodine (CsI) rather than as molecular iodine $\left(\mathrm{I}_{2}\right)$. Therefore, the CsI is selected as representative FPs.

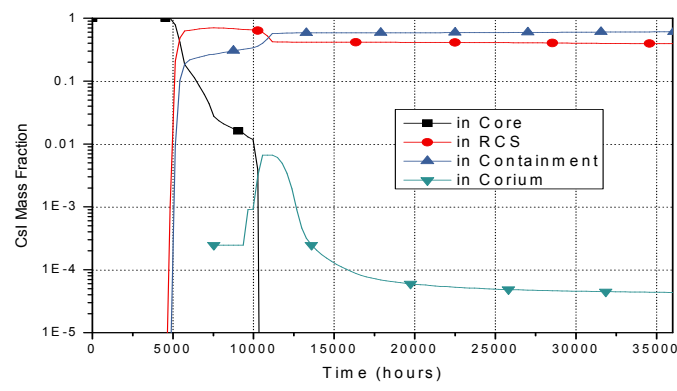

Fig. 1 CsI Distribution in Base Case Analysis
The behavior of CsI as a representative FP is illustrated in Fig 1 for the OPR-1000. The release of CsI into the RCS was initiated when the cladding damage occurred at 1.2 hours (4,320 seconds) after an accident initiation. Afterward all of the CsI have been released from the core to the RCS at 2.85 hours (10,260 seconds). Once the FP is released to the $\mathrm{RCS}$, it is transported to an atmosphere of the containment building $(\mathrm{C} / \mathrm{B})$ through a ruptured hole of the cold leg. About $40 \%$ and $60 \%$ of the initial CsI inventory are distributed in the RCS and $\mathrm{C} / \mathrm{B}$, respectively. During a melting process of the OPR-1000, the vertical fuel rods have an enough chance to be oxidized with high temperature. Accordingly, all the CsI were released to the RCS primarily from the core, and then moved to the containment partially. The mass fraction of CsI in the corium is about the order of magnitude of $10^{-4}$ $10^{-2}$. On the other hand there is no FP release to the environment for the OPR-1000 because the C/B had not failed during 72 hours of the calculation time.

\section{Uncertainty Analysis}

In severe accident analysis there are uncertainties in the physical phenomena. There are also uncertainties in the MAAP models. Users have control over the uncertainties via so-called 'model parameters', ${ }^{5)}$. They are either used as an input to a given physical model or to select between different physical models. This feature of the code architecture was included specifically to facilitate sensitivity or uncertainty analysis. In this study, input variables assigned as the model parameters to affect FP behaviors during a severe accident are identified, and their uncertainty is characterized using a user specified distribution. These parameters were selected based on MAAP input parameter files. A best-estimate value range is given for these parameters in the code documentation.

For the present uncertainty analysis, 13 inputs have been selected and the corresponding uncertainty distributions have been defined as shown in Table 2 . In order to propagate these uncertain inputs through the MAAP code, they were sampled using the LHS technique.

The results of the 200 MAAP analyses constitute samples of the distribution of the FP fraction given the uncertainties expressed in the Table 2. In this study, any dependency between parameters was not considered in the sampling process, and thus all parameters were treated as independent.

The results of all 200 LHS analyses of the uncertain code parameters are shown in Fig. 2 to Fig. 5. Since this application was focused on determining an estimate of the FP distribution in the core, the RCS, the molten corium (debris bed), and the CD/B of OPR-1000, the cesium iodine (CsI) fraction at the corresponding location has been figured out, respectively. There is not much change in distribution of CsI mass fraction after 10 hours $(36,000$ seconds); therefore the calculations are performed during 10 hours. 
Table 2. Summary of MAAP modeling parameters considered in this study to fission product behavior.

\begin{tabular}{|l|l|c|c|c|}
\hline $\begin{array}{c}\text { MAAP Uncertainty } \\
\text { Parameters }\left(x_{i}\right)\end{array}$ & \multicolumn{1}{|c|}{ Description } & $\begin{array}{c}\text { Most Likely } \\
\text { Value }\end{array}$ & Range & Distribution \\
\hline$x_{1}=$ FFPREL & Multiplier for in-core FP and inert aerosol release & 1.0 & {$[0.01,1.0]$} & Triangle \\
\hline$x_{2}=$ FCSIVP & Multiplier to vapor pressure of CsI for vapor-aerosol equilibrium & 1.0 & {$[0.01,1.0]$} & Triangle \\
\hline$x_{3}=$ FCSHVP & Multiplier to vapor pressure of CsOH for vapor-aerosol equilibrium & 0.1 & {$[0.01,1.0]$} & Loguniform \\
\hline$x_{4}=$ FVPREV & Multiplier to vapor pressures of CsI and CsOH for revaporization & 1.0 & {$[0.01,2.0]$} & Triangle \\
\hline$x_{5}=$ XRSEED & Initial seed radius for hygroscopic aerosol growth & $0.3 \mathrm{e}-6$ & {$[1.0 \mathrm{e}-7,3.0 \mathrm{e}-7]$} & Loguniform \\
\hline$x_{6}=$ FAERDC & Ratio of existing airborne aerosol mass to aerosol mass in steady-state & 8.0 & {$[1,100]$} & Loguniform \\
\hline$x_{7}=$ GSHAPE & Gamma shape factors to account for nonspherical aerosols & 2.5 & {$[1.0,10]$} & Loguniform \\
\hline$x_{8}=$ CSHAPE & Chi shape factors to account for nonspherical aerosols & 1.0 & {$[1.0,15]$} & Loguniform \\
\hline$x_{9}=$ FEO & Aerosol collision efficiency & 0,33 & {$[0.33,1.0]$} & Loguniform \\
\hline$x_{10}=$ FEFFDR & Containment spray aerosol capture efficiency & 0.02 & {$[0.01,0.05]$} & Loguniform \\
\hline$x_{11}=$ XRDB & Radius of aerosol particles released from debris beds into water pools & $0.01 \mathrm{e}-6$ & {$[1.0 \mathrm{e}-8,1.0 \mathrm{e}-6]$} & Loguniform \\
\hline$x_{12}=$ FDFDB & $\begin{array}{l}\text { Multipliers to decontamination factors for aerosol releases from debris } \\
\text { beds into water pools }\end{array}$ & 1.0 & {$[1.0 \mathrm{e}-4,100]$} & Loguniform \\
\hline$x_{13}=$ FDFJJ & $\begin{array}{l}\text { Multipliers to decontamination factors for aerosol releases from } \\
\text { containment junctions into water pools }\end{array}$ & 1.0 & {$[1.0 \mathrm{e}-4,100]$} & Loguniform \\
\hline
\end{tabular}

A CsI release from the core was initiated after a cladding damage occurred. From then on all of the released CsI have been transported to the RCS. Figure 2 shows that the samples of the distribution of CsI depletion time in core ranged between 9,300-10,500 seconds. The FP transported to the RCS is in turn moved to the $\mathrm{C} / \mathrm{B}$ through a ruptured hole of the cold leg or a reactor vessel breach. The samples of the distribution of CsI mass fraction in RCS ranged between 0.06 and 0.8 of initial inventory (see Fig. 3), and the mass fraction in $\mathrm{C} / \mathrm{B}$ brought this total to between 0.2 and 0.9 (see Fig. 4). Meanwhile the mass fraction of CsI remained in the corium is about $3 \times 10^{-4}-2 \times 10^{-2}$ of initial inventory (see Fig. 5).

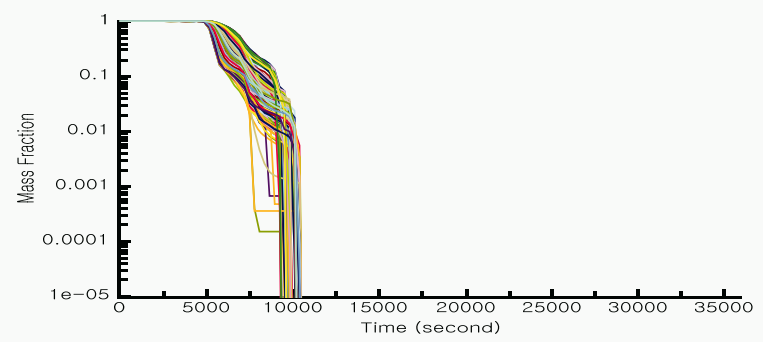

Fig. 2 Mass Fraction of CsI in Core for the Uncertainty Case

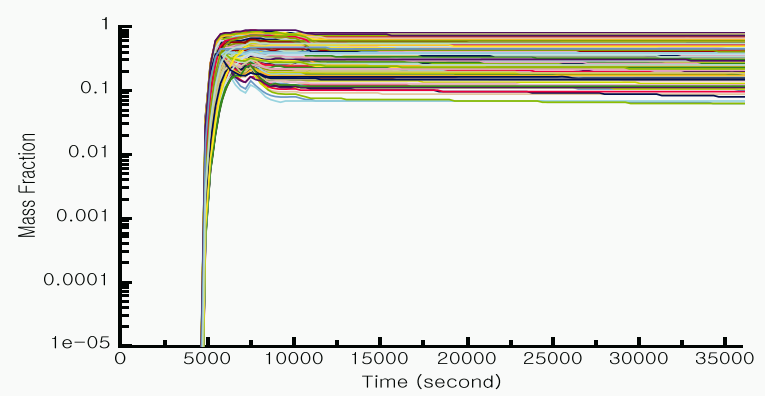

Fig. 3 Mass Fraction of CsI in RCS for the Uncertainty Case

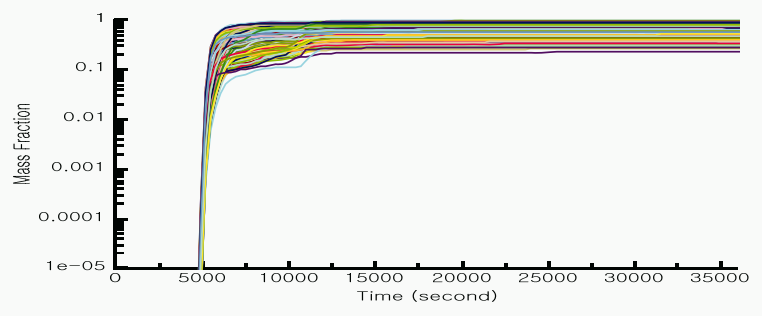

Fig. 4 Mass Fraction of CsI in C/B for the Uncertainty Case

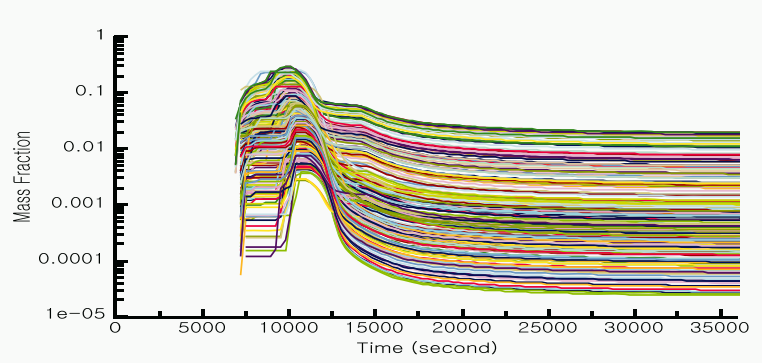

Fig. 5 Mass Fraction of CsI in Corium for the Uncertainty Case

In addition to characterizing the uncertainty distribution of predicted CsI release and retention, a type of linear regression analyses were also performed in order to identify which of the uncertain input parameters were most responsible for the uncertainty of the relevant output. As a result, two importance measures have been derived from the rank regression analysis for each output variable (CsI fraction in 4 locations of reactor and containment), i.e., partial rank correlation coefficients (PRCCs) and standardized rank regression coefficients (SRRCs) ${ }^{6)}$ 7). The results are summarized in Table 3 . 
As a result of regression analysis, the coefficients of determination, $\mathrm{R}^{2}$, were estimated to assess goodness-of-fits. As shown in Table 3, the estimated coefficients of determination are enough to rank dominant inputs to the corresponding outputs according to their relative magnitudes, from the statistical point of view. Based on Table 3, the model input 'FFPREL' is the key parameter to
CsI fraction in core (FCRT(2)) and in corium (FCMT(2)). In addition, three key parameters to FPST(2), a fraction of CsI in RCS has been identified as FFPREL, GSHAPE and CSHAPE. Whereas, FVPREV, GSHAPE and CSHAPE have the greatest impact on the retention of CsI in containment (FCOT(2)).

Table 3. Regression Analysis Results (No. of Samples: 200, Sampling Type: LHS)

\begin{tabular}{|l|c|c|c|c|c|c|c|c|}
\hline \multirow{2}{*}{$\begin{array}{l}\text { Input } \\
\text { Variables }\left(x_{i}\right)\end{array}$} & \multicolumn{2}{|c|}{ FCRT(2): } & \multicolumn{2}{c|}{ FPST(2): } & \multicolumn{3}{c|}{ FCOT(2): } & \multicolumn{2}{c|}{ FCMT(2): } \\
\cline { 2 - 9 } & CsI Fraction in Core & CsI Fraction in RCS & CsI Fraction in Containment & \multicolumn{2}{c|}{ CsI Fraction in Corium } \\
\hline$x_{1}=$ FFPREL & SRRC & PRCC & SRRC & PRCC & SRRC & PRCC & SRRC & PRCC \\
\hline$x_{2}=$ FCSIVP & $-\mathbf{0 . 9 0}$ & $\mathbf{- 0 . 9 0}$ & $\mathbf{0 . 3 6}$ & $\mathbf{0 . 8 4}$ & -0.10 & -0.25 & $\mathbf{- 0 . 9 9}$ & $\mathbf{- 0 . 9 8}$ \\
\hline$x_{3}=$ FCSHVP & -0.04 & 0.09 & -0.02 & -0.10 & -0.08 & -0.19 & 0.03 & 0.15 \\
\hline$x_{4}=$ FVPREV & -0.03 & -0.06 & -0.03 & -0.13 & $\mathbf{0 . 4 3}$ & $\mathbf{0 . 7 5}$ & -0.02 & -0.09 \\
\hline$x_{5}=$ XRSEED & 0.02 & 0.05 & 0.00 & -0.01 & -0.02 & -0.06 & -0.01 & -0.03 \\
\hline$x_{6}=$ FAERDC & -0.01 & -0.03 & 0.00 & -0.01 & 0.00 & 0.00 & 0.01 & 0.04 \\
\hline$x_{7}=$ GSHAPE & 0.00 & 0.01 & $\mathbf{0 . 6 3}$ & $\mathbf{0 . 9 4}$ & $\mathbf{- 0 . 5 9}$ & $\mathbf{- 0 . 8 4}$ & 0.01 & 0.04 \\
\hline$x_{8}=$ CSHAPE & 0.02 & 0.05 & $\mathbf{- 0 . 5 9}$ & $\mathbf{- 0 . 9 3}$ & $\mathbf{0 . 6 1}$ & $\mathbf{0 . 8 5}$ & 0.00 & 0.03 \\
\hline$x_{9}=$ FEO & 0.03 & 0.07 & 0.13 & 0.48 & -0.11 & -0.29 & 0.01 & 0.07 \\
\hline$x_{10}=$ FEFFDR & 0.01 & 0.03 & 0.01 & 0.03 & -0.01 & -0.02 & 0.01 & 0.04 \\
\hline$x_{11}=$ XRDB & 0.00 & 0.00 & -0.01 & -0.04 & 0.02 & 0.05 & 0.04 & 0.21 \\
\hline$x_{12}=$ FDFDB & 0.00 & -0.01 & -0.01 & -0.02 & 0.03 & 0.09 & 0.01 & 0.03 \\
\hline$x_{13}=$ FDFJJ & 0.02 & 0.05 & 0.02 & 0.10 & 0.00 & -0.01 & -0.02 & -0.08 \\
\hline$R^{2}$ ( N=200) & $\mathbf{0 . 8 3}$ & $\mathbf{0 . 8 3}$ & $\mathbf{0 . 9 5}$ & $\mathbf{0 . 9 5}$ & $\mathbf{0 . 8 6}$ & $\mathbf{0 . 8 6}$ & $\mathbf{0 . 9 7}$ & $\mathbf{0 . 9 7}$ \\
\hline
\end{tabular}

\section{Conclusions}

In this paper, a sampling-based uncertainty analysis has been performed to statistically quantify uncertainties associated with the FPs behavior, based on key modeling parameters employed in the MAAP code and LHS samples for those parameters. The accident sequence considered was a small LOCA expected in the OPR-1000 plant. As a result, uncertainties addressed in CsI fraction in 4 locations of reactor and containment has been quantified as a function of time.

In addition to characterizing uncertainties addressed in CsI fraction, a type of linear regression analyses were performed in order to identify which of the uncertain input parameters were most responsible for the uncertainty of the relevant output. As a result, two importance measures (i.e., PRCC and SRRC) have been derived for CsI fraction in each of 4 locations of reactor and containment. The ranks of estimated importance measures could be used to identify key areas where additional research may best be applied to further reduce the residual uncertainties of the predictions, and thereby better apply limited research resources.

\section{Acknowledgement}

This study has been carried out under the nuclear R\&D program planned by the Korean Ministry of Education, Science and Technology (MEST).

\section{References}

1) Fauske \& Associates, LLC, "MAAP4 Modular Accident Analysis Program for LWR Power Plants User's Manual," Project RP3131-02 (prepared for EPRI), May 1994-June 2005.

2) Gauntt, R.O., Cole, R.K., et al., "MELCOR Computer Code Manuals, Version 1.8.5," NUREG/CR-6119, 2005.

3) Gauntt, R.O, "Uncertainty Analyses Using the MELCOR Severe Accident Analysis Code," SNL, OECD Workshop Proceeding, Aix-en-Provence, November 2005

4) Ho G. LIM, Sang H. HAN, "Development of T/H Uncertainty Analysis S/W MOSAIQUE," KJPSA10, May 2009.

5) EPRI, "MAAP4 Applications Guidance. Palo Alto," CA: 2006. 1013500 .

6) Iman R.L, Helton J.C, Campbell J.E, “An approach to sensitivity analysis of computer models: Part I-introduction, input variable selection and preliminary variable assessment," J Qual Technol 13: 174-183. 1981.

7) Iman R.L, Helton J.C, Campbell J.E, “An approach to sensitivity analysis of computer models: Part II- ranking of input variables, response surface validation, distribution effect and technique synopsis," J Qual Technol 13: 232-240. 1981. 